\title{
Viscosity of Liquid Perfluoroalkanes and Perfluoroalkylalkane Surfactants
}

\author{
Pedro Morgado, ${ }^{\dagger}$ Carlos M. C. Laginhas, ${ }^{\ddagger}$ J. Ben Lewis, $^{\S}$ Clare McCabe, ${ }^{\S, \|}$ Luís F. G. Martins, ${ }^{*, \neq}$ and \\ Eduardo J. M. Filipe ${ }^{*+}$ \\ ${ }^{\dagger}$ Centro de Química Estrutural, Instituto Superior Técnico, 1049-001 Lisboa, Portugal \\ ${ }^{\ddagger}$ Centro de Química de Évora, Universidade de Évora, Rua Romão Ramalho, 59, 7000-671 Évora, Portugal \\ ${ }^{\S}$ Department of Chemical and Biomolecular Engineering and "Department of Chemistry, Vanderbilt University, Nashville, \\ Tennessee 37235, United States
}

\begin{abstract}
As part of a systematic study of the thermophysical properties of two important classes of fluorinated organic compounds (perfluoroalkanes and perfluoroalkylalkanes), viscosity measurements of four $n$-perfluoroalkanes and five perfluoroalkylalkanes have been carried out at atmospheric pressure and over a wide range of temperatures $(278-353 \mathrm{~K})$. From the experimental results the contribution to the viscosity from the $\mathrm{CF}_{2}$ and $\mathrm{CF}_{3}$ groups as a function of temperature have been estimated. Similarly, the contributions for $\mathrm{CH}_{2}$ and $\mathrm{CH}_{3}$ groups in $n$-alkanes have been determined using literature data.

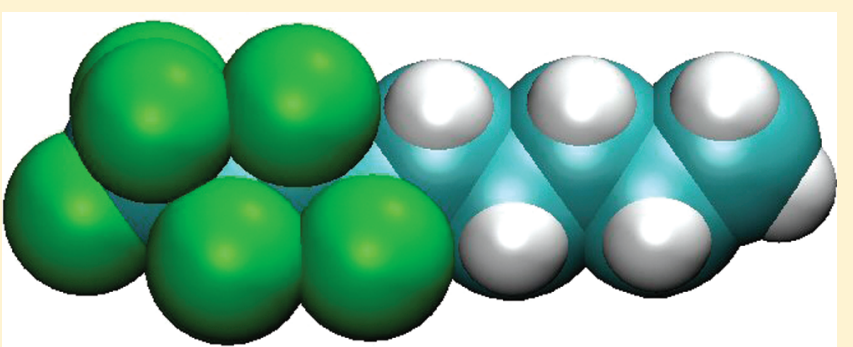
For perfluoroalkylalkanes, the viscosity results were interpreted in terms of the contributions of the constituent $\mathrm{CF}_{2}, \mathrm{CF}_{3}, \mathrm{CH}_{2}$, and $\mathrm{CH}_{3}$ groups, the deviations from ideality on mixing hydrogenated and fluorinated chains, and the contribution due to the formation of the $\mathrm{CF}_{2}-\mathrm{CH}_{2}$ bond. A standard empirical group contribution method (Sastri-Rao method) has also been used to estimate the viscosities of the perfluoroalkylalkanes. Finally, to obtain molecular level insight into the behavior of these molecules, all-atom molecular dynamics simulations have been performed and used to calculate the densities and viscosities of the perfluoroalkylalkanes studied. Although both quantities are underestimated compared to the experimental data, with the viscosities showing the largest deviations, the trends observed in the experimental viscosities are captured.
\end{abstract}

\section{INTRODUCTION}

The scientific interest in highly fluorinated hydrocarbons, because of their important and diversified applications, is a direct consequence of their unique properties in comparison with their hydrocarbon counterparts, such as inertness, low cohesive energy, nonflammability, structural rigidity, and low molecular density. As a result, fluorocarbons are used as high-performance lubricants, fire retardants, surfactants, surface coating films (to prevent adhesion), and solvents in biphasic synthesis. ${ }^{1,2}$ In this latter regard, the use of fluorinated alkanes as cosolvents in supercritical extraction or supercritical reaction media with $\mathrm{CO}_{2}{ }^{3,4}$ is a matter of special interest. It is, however, in the biomedical field that fluorocarbons find their most striking and exciting applications. Their biocompatibility and high mass density make them ideal liquids for eye surgery and in the treatment of burns. ${ }^{5,6}$ One of the most striking properties of fluorocarbons is their enhanced ability to solubilize gaseous substances, in particular, respiratory gases such as oxygen and carbon dioxide. This property, along with their biocompatibility, makes them obvious candidates to be used as active substances in emulsions of temporary blood substitutes (oxygen carriers in surgery or in the context of hemorrhagic shock) and as neat liquids in liquid ventilation for lung failure. In both cases, cyclic and aliphatic compounds whose molecular structure is based on perfluoroalkyl chains are used. ${ }^{7}$
Perfluoroalkanes constitute a very interesting chemical family not only because of their commercial applications but also for fundamental reasons. Their low cohesive energy reflects directly in higher vapor pressures and lower surface tensions when compared to alkanes with the same number of carbon atoms, despite the higher molecular weight. ${ }^{8}$ The molecular rigidity of perfluoroalkanes also contributes to the low molecular densities and high isothermal compressibilities observed. ${ }^{9}$ On the other hand, perfluoroalkanes are extremely hydrophobic with practically immeasurable solubility in water. ${ }^{7}$ Despite the structural resemblance of their components, binary mixtures of alkanes and perfluoroalkanes are highly nonideal, displaying liquid-liquid immiscibility in extensive ranges of temperature and pressure. ${ }^{10}$ Perfluoroalkanes are, thus, not only hydrophobic but also "hydrocarbon-phobic". Being immiscible with both aqueous and organic solvents, perfluoroalkanes can be thought of as an alternative media, opening ways to new industrial applications.

Mixtures of alkanes and perfluoroalkanes have been extensively studied both experimentally and theoretically since the 1950 's. ${ }^{11}$ Some of their characteristics are: large and positive

Received: February 11, 2011

Revised: $\quad$ May 11, 2011

Published: June 02, 2011 
values of the excess molar Gibbs energy, $G_{\mathrm{m}}{ }^{\mathrm{E}}$ (between 1000 and $\left.2000 \mathrm{~J} \cdot \mathrm{mol}^{-1}\right) ; 12,13$ large positive values of $V_{\mathrm{m}}^{\mathrm{E}}$, around $5 \mathrm{~cm}^{3} \cdot \mathrm{mol}^{-1}$, which are among the largest known for nonelectrolyte solutions; ${ }^{14}$ and large liquid-liquid immiscibility gaps. The upper critical solution temperature (UCST) for this kind of systems increases with the chain length of both the alkane and the perfluoroalkane, being more sensitive to increases in the alkylic chain. ${ }^{15}$ Further indication of the anomalous behavior of systems involving alkanes and perfluoroalkanes is given by the partial molar volumes at infinite dilution of $n$-alkanes in perfluoroalkanes and vice versa. ${ }^{14-16}$ From these results it can be concluded that the volume of perfluoroalkanes increases by $\sim 13 \%$ when dissolved in $n$-alkanes at infinite dilution, whereas for $n$-alkanes dissolved in perfluoroalkanes, the volume increases by $\sim 20 \%$.

Most theories of liquids have failed in predicting the unexpected phase behavior displayed by (alkane + perfluoroalkane) systems and an extremely weak unlike interaction between alkane and perfluoroalkane molecules has been suggested. ${ }^{17} \mathrm{McC}$ abe et al., ${ }^{18}$ using a version of the statistical associating fluid theory for potential of variable range (SAFT-VR), were able to describe the high pressure phase behavior and critical lines of binary systems of alkanes and perfluoroalkanes, using a binary interaction parameter that corresponded to a $8 \%$ decrease in the cross (or unlike) interaction energy (in comparison with the geometric mean prediction). ${ }^{10}$ This binary parameter was fitted to experimental data from (butane + perfluorobutane) and used to predict the phase behavior in the other systems studied in a transferable way. More recently, Morgado et al. ${ }^{19}$ in a related study succeed in predicting composition coexistence curves, excess molar volumes, and UCST for mixtures involving alkanes and perfluoroalkanes (between $\mathrm{C}_{5}$ and $\mathrm{C}_{8}$ near room temperature) using SAFT-VR. A different binary interaction parameter was needed, fitted in this case to LLE equilibrium and UCST of the system ( $n$-hexane + perfluorohexane), and, as in the previous work, was used in a transferable way. Studies of alkane + perfluoroalkane systems with other versions of the SAFT equation have shown similar behavior, in that a reduction in the strength of the cross interaction energy predicted by the geometric mean is needed to accurately describe the phase behavior. ${ }^{20-24}$

While alkanes have been very widely studied in the literature by molecular simulation and are typically the focus of force field development work, a more limited number of simulation studies have considered perfluoroalkanes and their binary mixtures with alkanes in an effort to further understand the behavior of these systems. ${ }^{10,25-33}$ In particular, Song et al. ${ }^{10}$ calculated cross second virial coefficients, gas-liquid solubilities, and enthalpies of mixing for binary mixtures of $n$-alkanes and perfluoroalkanes by computer simulation using the OPLS-AA force field. Agreement between simulation and experiment was only possible with the introduction of corrections to geometric mean rule, reducing by $25 \%$ the $\mathrm{H}-\mathrm{F}$ energetic interaction, which, according to the authors, corresponds to an overall $10 \%$ reduction of the cross energetic interaction.

Given the mutual antipathy between alkyl and perfluoroalkyl chains, semifluorinated alkanes (also known as perfluoroalkylalkanes (PFAA) or alkyl-perfluoroalkyl diblocks) can be considered amphiphiles toward these two media, thus opening a myriad of possibilities in terms of both research and industrial applications. For example, aggregation in solvents selective for one of the blocks, the formation of micelles and vesicles, ${ }^{34,35}$ the observation of smectic liquid crystalline phases, ${ }^{36,37}$ the formation of nanoscale patterns in molecular films of either pure or mixed perfluoroalkylalkanes, ${ }^{38,39}$ and organization in the solid state into layered structures, have all been reported. ${ }^{40-43}$ For the same reasons, semifluorinated alkanes can also play an important role in stabilizing blood substitute emulsions. Stability is one of the most important characteristics of these microheterogeneous systems, so that they can be used in biomedical applications. ${ }^{44}$ The disruption of perfluorocarbon (PFC)-in-water emulsions by Ostwald ripening (molecular diffusion) depends on the solubility and diffusion coefficient of the active agent (usually perfluorooctyl bromide) in water, among other properties. ${ }^{7}$ It is a common practice to add to the emulsion a heavier perfluorocarbon to reduce emulsion decaying by molecular diffusion (adjuvant). Apart from the emulsifier (usually a natural phospholipid or a fluorinated chain surfactant), PFC-in-water emulsions usually contain cosurfactants to stabilize the emulsion. Perfluoroalkylalkanes are one of the most promising cosurfactants for this type of emulsions. ${ }^{45}$

Another promising type of PFC/PFAA-based organized systems for gas transport and ultrasound imaging contrast is microbubbles of gases in water. ${ }^{46-49}$ The gaseous microbubbles (saturated in PFC, usually, a light liquid perfluoroalkane) are trapped in capsules made of rigid multilayers of polyelectrolytes, polymers, crystallized lipids, or flexible surfactants (mainly phospholipids). ${ }^{50}$ These gas bubbles usually have short lives in the intravascular medium. PFCs as filling gases are effective in increasing the microbubble persistence, because of low solubility and diffusion coefficient of PFCs in the continuous aqueous phase. ${ }^{51}$ It seems likely that light perfluoroalkylalkanes can be more effectively used as filling gas within the aqueous microbubbles.

Despite their interesting behavior and vast potential, very little work has been done experimentally to characterize the thermodynamic behavior of PFAAs in the liquid state, either pure or mixed with other substances. Perhaps the first such studies were those of de Loos et al., ${ }^{52}$ in which the phase envelope of binary and quasi-binary mixtures of the simplest PFAA, $\mathrm{CF}_{3} \mathrm{CH}_{3}$, with the linear alkanes undecane, dodecane, and tridecane and their binary mixtures, was determined, and the work of Tochigi et al. ${ }^{53}$ in which the vapor-liquid equilibrium of liquid mixtures of perfluorobutylethane and octane at $101.3 \mathrm{kPa}$ was measured. More recently, we have performed a systematic study of the thermophysical properties of several PFAAs. In particular, densities as a function of temperature and pressure were measured for $\mathrm{F} 6 \mathrm{H} 6$ and $\mathrm{F} 6 \mathrm{H} 8^{54}$ and for F4H5, F4H6, and F4 $48 .^{55}$ The results were interpreted in terms of the volumes of the constituent hydrogenated and perfluorinated segments corrected for the corresponding excess volumes and the volume contribution of the $\mathrm{CH}_{2}-\mathrm{CF}_{2}$ junction. A heteronuclear version of the SAFT-VR equation of state was used to model these systems. ${ }^{56,57}$ The theory was able to reproduce the experimental molar volumes with reasonable accuracy but failed to predict the thermal expansivities and isothermal compressibilities. ${ }^{48,49}$ We have also determined the partial molal volumes for a series of perfluoroalkanes (with 5, 6, 8, and 9 carbon atoms) and perfluoroalkylalkanes (F4H5, F4H6, F4H8, F6H6, $\mathrm{F} 6 \mathrm{H} 8, \mathrm{~F} 10 \mathrm{H} 8$, and F8H18) in $n$-octane at $25^{\circ} \mathrm{C} \cdot{ }^{15,16}$ It was found that for perfluoroalkanes the partial molar volumes at infinite dilution were $13 \%$ higher than the corresponding pure molar volumes, whereas for PFAAs this increment is approximately 5\%. Again, the results were rationalized in terms of the partial molar volumes at infinite dilution of the corresponding hydrogenated and perfluorinated segments and the contribution from the $\mathrm{CH}_{2}-\mathrm{CF}_{2}$ link. It was found that contribution to the volume of the diblock junction is independent of chain length of the hydrogenated segment but decreases with the chain length of the fluorinated segment. 
PFAAs have been the subject of several simulation studies to determine, for example, the structure of the liquid interface of pure perfluorocarbon-hydrocarbon diblocks, ${ }^{58}$ their aggregation, ${ }^{59}$ and liquid crystalline behavior. ${ }^{60}$ Of perhaps the most relevance to the current work, Pádua and co-workers performed all-atom molecular dynamics simulations of liquid perfluorooctylethane, perfluorohexylethane, and perfluorohexylhexane using the OPLS force field to calculate liquid densities, vaporization enthalpies, and the solubility of oxygen, carbon dioxide, and water. ${ }^{61,62}$ To describe the PFAA a new cross-torsional term was proposed; however, given the scarcity of experimental data at that time, only density data at a single state point were available to compare with the simulation results. The solubility of water in several PFAA has since been measured and for F6H6 found to be $\sim 500$ times higher than that predicted by the simulations. ${ }^{63}$ In subsequent work Grest and co-workers ${ }^{64}$ studied the densities and surface tensions of a range of alkanes, perfluoroalkanes and PFAA using the modified OPLS potential proposed by Pádua $^{61}$ and the reduced $\mathrm{H}-\mathrm{F}$ interaction proposed by Song et al. ${ }^{10}$

While much of the previous work on perfluoroalkanes has focused on equilibrium properties, transport properties are less well-characterized, yet the viscosity for example is a key property in view of their applications. Alkanes and perfluoroalkanes display very different viscosities (e.g., at $25^{\circ} \mathrm{C}$ the viscosity of $n$-hexane is $0.30 \mathrm{mPa} \cdot \mathrm{s}$ while that of perfluorohexane is 0.64 $\mathrm{mPa} \cdot \mathrm{s})$. Perfluoroalkylalkanes are, thus, expected to show intermediate values of viscosity, depending on the total chain length of the molecule and the relative proportion of alkylic and perfluoroalkylic segments. Experimental data on the viscosity of fluorocarbons are rather scarce in literature. In perhaps the only study to date, Freire et al. ${ }^{65}$ have measured the viscosity of several linear (F6 to F9), cyclic, aromatic, and $\alpha$-substituted perfluorocarbons, over a relatively limited range of temperature.

In this work, the viscosity of four perfluoroalkanes (perfluoropentane, perfluorohexane, perfluorooctane, and perfluorononane) and five PFAAs (perfluorobutylpentane, perfluorobutylhexane, perfluorobutyloctane, perfluorohexylhexane, and perfluorohexyloctane) were measured in a large range of temperatures, from 278 to $353 \mathrm{~K}$. Following the procedure adopted in previous work, the viscosity of the PFAAs was estimated from the contributions to the viscosity due to the $\mathrm{CF}_{3}, \mathrm{CF}_{2}, \mathrm{CH}_{2}$, and $\mathrm{CH}_{3}$ groups. The differences found between the calculated and the experimental results are rationalized in terms of the contribution of the $\mathrm{CH}_{2}-\mathrm{CF}_{2}$ bond and the deviations from ideality of mixtures of $n$-alkanes and perfluoroalkanes. The viscosity and density of all PFAAs were also calculated by molecular dynamics simulation. In a previous work McCabe et al. ${ }^{29,30}$ predicted the viscosities of pure perfluoroalkanes ( $\mathrm{F} 4$ to F7) by molecular dynamics simulation and determined that while a united atom force field underestimates the viscosity of alkanes (see for example refs 66-68) they can be used to reliably predict trends in the viscosity; however, for perfluoroalkanes, the increased molecular roughness due to the size of the $\mathrm{F}$ atom compared to the $\mathrm{H}$ atom results in the need for all-atom simulations to capture the experimental behavior. In this work we have therefore used an all-atom force field to study the PFAAs. Finally, the Sastri-Rao empirical group contribution method ${ }^{80}$ was also used to estimate the viscosities of the semifluorinated alkanes studied.

\section{EXPERIMENTAL SECTION}

Materials. Perfluorobutylpentane (F4H5), perfluorobutylhexane (F4H6), perfluorobutyloctane (F4H8), perfluorohexylhexane (F6H6), and perfluorohexyloctane (F6H8) were purchased from
Fluoron GMBH as ultrapurified chemicals with claimed purity of $100 \%$. The purity of these compounds was checked by ${ }^{19} \mathrm{~F}$ and ${ }^{1} \mathrm{H}$ NMR spectra in a $500 \mathrm{MHz}$ Bruker spectrometer, and less than $1 \%$ of impurities was detected. Hence, these compounds were used without further purification. Perfluoropentane (F5) and perfluorononane (F9) were obtained from Apollo Scientific, with 97\% ( $85 \% n$-isomer) and 99\% purities, respectively; perfluorohexane (F6, 99\%) and perfluorooctane (F8, 98\%) were obtained from Aldrich. All were used as received.

Procedure. The experimental viscosity measurements were all carried out at atmospheric pressure and in the temperature range from 298 to $353 \mathrm{~K}$ for semifluorinated alkanes as well as for perfluorononane. For smaller perfluoroalkanes (perfluoropentane to perfluorooctane) and F6H6, temperatures of 278 and $288 \mathrm{~K}$, respectively, were reached for the temperature range minimum; however, because of their lower boiling points viscosity measurements were extended only to $297 \mathrm{~K}$ for perfluoropentane and $323 \mathrm{~K}$ for perfluorohexane.

The kinematic viscosities were measured using Schott-Geräte Ubbelhode viscometers with an automatic measuring unit AVS 440. The Ubbelhode viscometer type 545-00/0 was used for viscosity measurements, except for $\mathrm{F} 6 \mathrm{H} 8$ and the lowest temperatures for $\mathrm{F} 6 \mathrm{H} 6$ for which a 545-03/0c viscometer was employed. The viscosity measuring system comprises a viscometer stand with optical sensors (AVS/S), an automatic pumping system, and a control and recording unit; the viscometer stand is immersed in a thermostatic bath, with a temperature stability better than $0.01 \mathrm{~K}$. Each flow time reported is the average of five independent measurements, with a scattering of less than $0.2 \%$. The uncertainty of each flow time measurement using this unit is $0.01 \mathrm{~s}$, and the overall uncertainty of viscosity measurements was estimated to be less than $0.8 \%$. The temperature was measured with a platinum resistance probe coupled with a $51 / 2$ digital multimeter (Keithley 191 ), with an accuracy of $0.05 \mathrm{~K}$ and a precision of $0.01 \mathrm{~K}$.

As ancillary data for dynamic viscosity calculation, atmospheric pressure densities were measured for all compounds studied using an Anton Paar DMA 5000 vibrating-tube densimeter. The instrument was calibrated with water (distilled, deionized in a Milli-Q185 Plus water purification system and freshly boiled) and air at $20.000{ }^{\circ} \mathrm{C}$, taking into account atmospheric pressure. This densimeter has an internal temperature control system, which is stable at $T \pm 0.001 \mathrm{~K}$.

\section{SIMULATION DETAILS}

The optimized potentials for liquid simulations all-atom (OPLS-AA) force field ${ }^{69}$ with the extension to perfluoroalkanes by Watkins and Jorgensen ${ }^{70}$ has been used to describe the PFAAs. The cross-dihedral terms between the fluorinated and the hydrogenated side of the PFAA molecules was described using the torsional parameters proposed by Pádua. ${ }^{61}$ In the OPLS forcefield a Lennard-Jones potential describes the intermolecular interactions and the intramolecular interactions between sites separated by three or more bonds. Since good agreement with experimental data has been obtained in the literature for the density of PFAAs when geometric mean values are used to calculate the strength of the cross interaction between the $\mathrm{H}$ and $\mathrm{F}$ atoms, ${ }^{63}$ in this initial study simple geometric combining rules were used to determine the cross interactions. Bond stretching and bond angle bending are described by harmonic potentials and torsional motion characterizing the preferred orientational and rotational barriers around all nonterminal bonds is described through the potentials of Jorgensen and Pádua. 
Table 1. Polynomial Coefficients for Fitting Atmospheric Densities as a Function of Temperature to the Equation $\rho=a_{0}+a_{1} T+$ $a_{2} T^{2}+a_{3} T^{3}$

\begin{tabular}{lccrcc} 
compound & $a_{3}$ & $a_{2}$ & $a_{1}$ & $a_{0}$ & std dev \\
F5 & $-3.3333 \times 10^{-8}$ & $2.3144 \times 10^{-5}$ & $-8.2832 \times 10^{-3}$ & 2.9063 & $3.11 \times 10^{-6}$ \\
F6 & $-1.7835 \times 10^{-8}$ & $1.1812 \times 10^{-5}$ & $5.2827 \times 10^{-3}$ & 2.6679 & $4.08 \times 10^{-5}$ \\
F8 & $-1.2809 \times 10^{-8}$ & $9.3084 \times 10^{-6}$ & $-4.7012 \times 10^{-3}$ & 2.6694 & $1.32 \times 10^{-5}$ \\
F9 & $-8.1381 \times 10^{-9}$ & $5.5389 \times 10^{-6}$ & $-3.5807 \times 10^{-3}$ & 2.5778 & $9.28 \times 10^{-5}$ \\
F4H5 & $-5.8741 \times 10^{-9}$ & $-4.3979 \times 10^{-6}$ & $-2.7101 \times 10^{-3}$ & 1.8597 & $1.11 \times 10^{-5}$ \\
F4H6 & $-4.5214 \times 10^{-9}$ & $3.4067 \times 10^{-6}$ & $-2.3608 \times 10^{-3}$ & 1.7785 & $1.22 \times 10^{-5}$ \\
F4H8 & $-3.3828 \times 10^{-9}$ & $2.7204 \times 10^{-6}$ & $-2.0863 \times 10^{-3}$ & 1.6790 & $1.05 \times 10^{-5}$ \\
F6H6 & $-4.6234 \times 10^{-9}$ & $3.7840 \times 10^{-6}$ & $-2.6309 \times 10^{-3}$ & 1.9569 & $1.15 \times 10^{-5}$ \\
F6H8 & $-5.0693 \times 10^{-9}$ & $4.5768 \times 10^{-6}$ & $-2.8456 \times 10^{-3}$ & 1.9058 \\
\hline
\end{tabular}

All simulations were performed within the NVT ensemble at densities obtained from NPT simulations at atmospheric pressure and $298.15 \mathrm{~K}$. All simulations were performed using the LAMMPS molecular dynamics code. ${ }^{71}$ A multiple time step technique was used to integrate the equations of motion with all of the intramolecular interactions treated as fast ( $0.1 \mathrm{fs})$ motions and the intermolecular interactions as slow ( $1 \mathrm{fs}$ ) motions. ${ }^{72}$ The simulations were performed with 243 molecules in a cubic box and a spherical potential cutoff of $10 \AA$.

The viscosity $\eta$ was calculated via the Green-Kubo formula from the integral of the stress-stress autocorrelation functions determined during the simulation, namely, ${ }^{73}$

$$
\eta=\frac{V}{k_{\mathrm{B}} T} \int_{0}^{\infty}\left\langle P_{\alpha \beta}(0) P_{\alpha \beta}(t)\right\rangle \mathrm{d} t
$$

where $V$ is the volume of the system, $k_{\mathrm{B}}$ is Boltzmann's constant, $T$ is temperature, and $t$ is time. The quantity $P_{\alpha \beta}(t)$ is the value of the $\alpha \beta$ off-diagonal component $(\alpha \beta=x, y, z)$ of the traceless symmetric stress tensor at time $t$, and so $P_{\alpha \beta}(t) P_{\alpha \beta}(0)$ is the stress - stress autocorrelation function and $\left\langle P_{\alpha \beta}(t) P_{\alpha \beta}(0)\right\rangle$ is its ensemble average (indicated by \langle\rangle ) measured during the course of the simulation. The simulations were run until a plateau was observed in the averaged correlation function and the viscosity and error calculated during the plateau region using block averaging. ${ }^{74}$ A correlation spacing of $10 \mathrm{fs}$ was used for all calculations and the total simulation time was $16 \mathrm{~ns}$ for F4H5, $28 \mathrm{~ns}$ for F4H6, 50 ns for F4H8, 70 ns for $\mathrm{F} 6 \mathrm{H} 6$, and 90 ns for $\mathrm{F} 6 \mathrm{H} 8$. To determine the appropriate length for each simulation the rotational relaxation time was calculated and each system run for a minimum of 100 multiples of the relaxation time, as discussed by Mondello and Grest $^{75}$ and Gordon. ${ }^{76}$ As an additional check, averages were taken over successively longer simulation times (up to the maximum values listed above) to ensure that a negligible change in the viscosity estimate was being observed. The rotational relaxation time of each molecule was estimated from the autocorrelation function of the molecular end-to-end vector,

$$
R(t)=\left\langle\frac{1}{N} \sum_{i}^{N} r_{i}(0) r_{i}(t)\right\rangle
$$

where $r_{i}$ is the end-to-end distance of molecule $i$ and the summation is over all $\mathrm{N}$ molecules in the system.

\section{RESULTS AND DISCUSSION}

The kinematic viscosities were measured for the perfluoroalkylalkanes in the temperature range from $\sim 298 \mathrm{~K}$ to $\sim 353 \mathrm{~K}$, except for F6H6 where measurements were made between $\sim 288$ $\mathrm{K}$ and $\sim 353 \mathrm{~K}$. For perfluoroalkanes, viscosities were measured in the range $\sim 278 \mathrm{~K}$ to $\sim 353 \mathrm{~K}$ for $\mathrm{F} 8$ and $\mathrm{F} 9$, in the range $278-323 \mathrm{~K}$ for F6 and in the range $278-297 \mathrm{~K}$ for F5. Liquid densities were also determined within the same temperature range, and both properties were measured at atmospheric pressure. The density results as a function of temperature were fitted to third-degree polynomials, which are reported in Table 1. From the measured kinematic viscosities and densities, dynamic viscosities were obtained at all temperatures for each compound and are presented at Table 2. The dynamic viscosities as a function of temperature were fitted to Andrade's equation:

$$
\ln \eta=A+\frac{B}{T}
$$

An Arrhenius-like behavior for the temperature dependence of viscosity is frequently assumed, where the resulting coefficients of eq $3, A$ and $B$, are identified with the logarithm of the preexponential factor $\left(\eta_{0}\right)$ and the activation energy divided by the ideal gas constant $\left(E_{\eta} / R\right)$, respectively. These parameters are presented in Table 3. It is found that both the activation energy and the pre-exponential factor increase with the chain length of the molecule and the fraction of fluorinated segments. The experimental points and the fitting curves are displayed in Figure 1, part a for perfluoroalkanes and part $b$ for perfluoroalkylalkanes. The experimental results for the perfluoroalkanes are compared with literature data in Figure 2. As can be seen, our viscosity results for F8 and F9 compare favorably with those of Freire et al. ${ }^{58}$ For F6, however, our results deviate $10-16 \%$ from those of the same authors but, in contrast, agree well with those from Stiles and Cady, ${ }^{77}$ with deviations of $2-5 \%$. As far as we are aware, no experimental viscosity data for the perfluoroalkylalkanes used in this work have been reported in literature.

The experimental results for the perfluoroalkanes and semifluorinated compounds follow some simple trends as discussed below. For perfluoroalkanes, a linear relation between $\ln \eta$ and the chain length can be seen in Figure $3 \mathrm{a}$ for a single interpolated temperature. For perfluoroalkylalkanes, $\ln \eta$ versus chain length is proportional to the number of "hydrogenated" carbon atoms, at constant number of "fluorinated" carbons and vice versa, as can be seen in Figure $3 b-d$ at three different temperatures. The results also show that viscosities of perfluoroalkylalkanes are intermediate between $n$-alkanes and perfluoroalkanes when 
Table 2. Kinematic and Dynamic Viscosities for All of the Studied PFA and PFAA

\begin{tabular}{|c|c|c|c|c|c|}
\hline \multirow{2}{*}{\multicolumn{3}{|c|}{ Studied PFA and PFAA }} & \\
\hline & & & $T / \mathrm{K}$ & $10^{7} \mathrm{v} / \mathrm{m}^{2} \cdot \mathrm{s}^{-1}$ & $\eta / \mathrm{mPa} \cdot \mathrm{s}$ \\
\hline$T / K$ & $10^{7} \mathrm{v} / \mathrm{m}^{2} \cdot \mathrm{s}^{-1}$ & $\eta / \mathrm{mPa} \cdot \mathrm{s}$ & 337.72 & 4.704 & 0.5738 \\
\hline & & & 342.70 & 4.453 & 0.5392 \\
\hline & F5 & & 347.70 & 4.220 & 0.5074 \\
\hline 278.09 & 3.710 & 0.6217 & 352.69 & 4.008 & 0.4782 \\
\hline 283.07 & 3.475 & 0.5768 & & F4H6 & \\
\hline 288.05 & 3.262 & 0.5363 & 297.95 & 10.25 & 1.289 \\
\hline 293.01 & 3.066 & 0.4991 & 302.94 & 9.449 & 1.181 \\
\hline \multirow[t]{2}{*}{296.97} & 2.921 & 0.4716 & 307.91 & 8.742 & 1.086 \\
\hline & F6 & & 312.75 & 8.125 & 1.004 \\
\hline 278.09 & 5.040 & 0.8712 & 317.84 & 7.522 & 0.9231 \\
\hline 283.06 & 4.675 & 0.8016 & 322.80 & 7.023 & 0.8563 \\
\hline 288.04 & 4.378 & 0.7443 & 327.76 & 6.575 & 0.7965 \\
\hline 292.90 & 4.102 & 0.6915 & 332.75 & 6.171 & 0.7427 \\
\hline 297.87 & 3.819 & 0.6381 & 337.68 & 5.805 & 0.6941 \\
\hline 297.90 & 3.839 & 0.6416 & 342.67 & 5.475 & 0.6502 \\
\hline 302.99 & 3.608 & 0.5973 & 347.66 & 5.172 & 0.6100 \\
\hline 307.86 & 3.321 & 0.5449 & 352.65 & 4.895 & 0.5733 \\
\hline 312.82 & 3.130 & 0.5088 & & F4H8 & \\
\hline 317.86 & 2.924 & 0.4705 & 297.90 & 16.48 & 1.993 \\
\hline \multirow[t]{2}{*}{322.77} & 2.830 & 0.4510 & 302.97 & 14.91 & 1.793 \\
\hline & F8 & & 307.82 & 13.57 & 1.623 \\
\hline 278.12 & 10.31 & 1.862 & 312.78 & 12.45 & 1.480 \\
\hline 283.10 & 9.321 & 1.672 & 317.75 & 11.50 & 1.359 \\
\hline 288.07 & 8.441 & 1.504 & 322.81 & 10.60 & 1.246 \\
\hline 293.13 & 7.689 & 1.360 & 327.78 & 9.833 & 1.149 \\
\hline 298.10 & 7.056 & 1.239 & 332.76 & 9.174 & 1.065 \\
\hline 303.06 & 6.494 & 1.132 & 337.72 & 8.562 & 0.9883 \\
\hline 308.02 & 6.004 & 1.039 & 342.70 & 8.088 & 0.9279 \\
\hline 313.02 & 5.568 & 0.9561 & 347.71 & 7.503 & 0.8555 \\
\hline 317.94 & 5.182 & 0.8829 & 352.70 & 7.044 & 0.7981 \\
\hline 322.91 & 4.835 & 0.8175 & & F6H6 & \\
\hline 327.86 & 4.523 & 0.7587 & 288.02 & 22.14 & 3.106 \\
\hline 332.83 & 4.243 & 0.7059 & 292.98 & 19.36 & 2.701 \\
\hline 337.87 & 3.984 & 0.6572 & 297.93 & 17.21 & 2.387 \\
\hline 342.86 & 3.749 & 0.6132 & 302.89 & 15.48 & 2.134 \\
\hline 347.84 & 3.532 & 0.5727 & 307.84 & 14.02 & 1.921 \\
\hline \multirow[t]{2}{*}{352.82} & 3.328 & 0.5347 & 307.96 & 14.01 & 1.921 \\
\hline & F9 & & 312.81 & 12.74 & 1.735 \\
\hline 297.92 & 9.982 & 1.784 & 317.78 & 11.63 & 1.576 \\
\hline 302.88 & 9.078 & 1.612 & 322.75 & 10.71 & 1.443 \\
\hline 307.84 & 8.293 & 1.462 & 327.72 & 9.849 & 1.318 \\
\hline 312.80 & 7.570 & 1.325 & 332.70 & 9.111 & 1.212 \\
\hline 312.91 & 7.563 & 1.324 & 337.77 & 8.451 & 1.117 \\
\hline 317.88 & 6.935 & 1.205 & 342.75 & 7.868 & 1.034 \\
\hline 322.85 & 6.408 & 1.106 & 347.66 & 7.334 & 0.9574 \\
\hline 327.81 & 5.942 & 1.018 & 352.64 & 6.860 & 0.8898 \\
\hline 332.79 & 5.530 & 0.9399 & & F6H8 & \\
\hline 337.76 & 5.157 & 0.8699 & 297.94 & 26.30 & 3.498 \\
\hline 342.73 & 4.821 & 0.8068 & 302.90 & 23.30 & 3.082 \\
\hline 347.73 & 4.515 & 0.7497 & 307.85 & 20.80 & 2.737 \\
\hline \multirow[t]{2}{*}{352.70} & 4.240 & 0.6983 & 307.86 & 20.80 & 2.736 \\
\hline & F4H5 & & 312.83 & 18.69 & 2.445 \\
\hline 297.89 & 7.942 & 1.022 & 317.79 & 16.89 & 2.197 \\
\hline 302.85 & 7.358 & 0.9412 & 322.76 & 15.34 & 1.985 \\
\hline 307.90 & 6.849 & 0.8703 & 327.82 & 13.99 & 1.799 \\
\hline 312.79 & 6.390 & 0.8068 & 332.69 & 12.85 & 1.644 \\
\hline 317.75 & 5.983 & 0.7504 & 337.67 & 11.83 & 1.505 \\
\hline 322.71 & 5.616 & 0.6996 & 342.63 & 10.94 & 1.177 \\
\hline 327.79 & 5.277 & 0.6527 & 347.73 & 10.13 & 1.273 \\
\hline 332.75 & 4.978 & 0.6115 & 352.72 & 9.43 & 1.177 \\
\hline
\end{tabular}

Table 2. Continued 
Table 3. Pre-exponential Factor $\left[\ln \left(\boldsymbol{\eta}_{0}\right)\right]$ and Activation Energy Divided by Ideal Gas Constant $\left(E_{\eta} / R\right)$ along with Their Standard Deviations $(\sigma)$

\begin{tabular}{llccc} 
& $\ln \left(\eta_{0}\right)$ & $\sigma\left[\ln \left(\eta_{0}\right)\right]$ & $\left(E_{\eta} / R\right) / \mathrm{K}$ & $\sigma\left(E_{\eta} / R\right)$ \\
F5 & -4.82 & 0.02 & 1208 & 7 \\
F6 & -5.00 & 0.06 & 1353 & 19 \\
F8 & -5.24 & 0.02 & 1626 & 5 \\
F9 & -5.46 & 0.03 & 1796 & 8 \\
F4H5 & -4.858 & 0.008 & 1453 & 2 \\
F4H6 & -4.98 & 0.01 & 1557 & 5 \\
F4H8 & -5.15 & 0.04 & 1736 & 13 \\
F6H6 & -5.60 & 0.06 & 1927 & 18 \\
F6H8 & -5.75 & 0.05 & 2081 & 17 \\
\hline
\end{tabular}
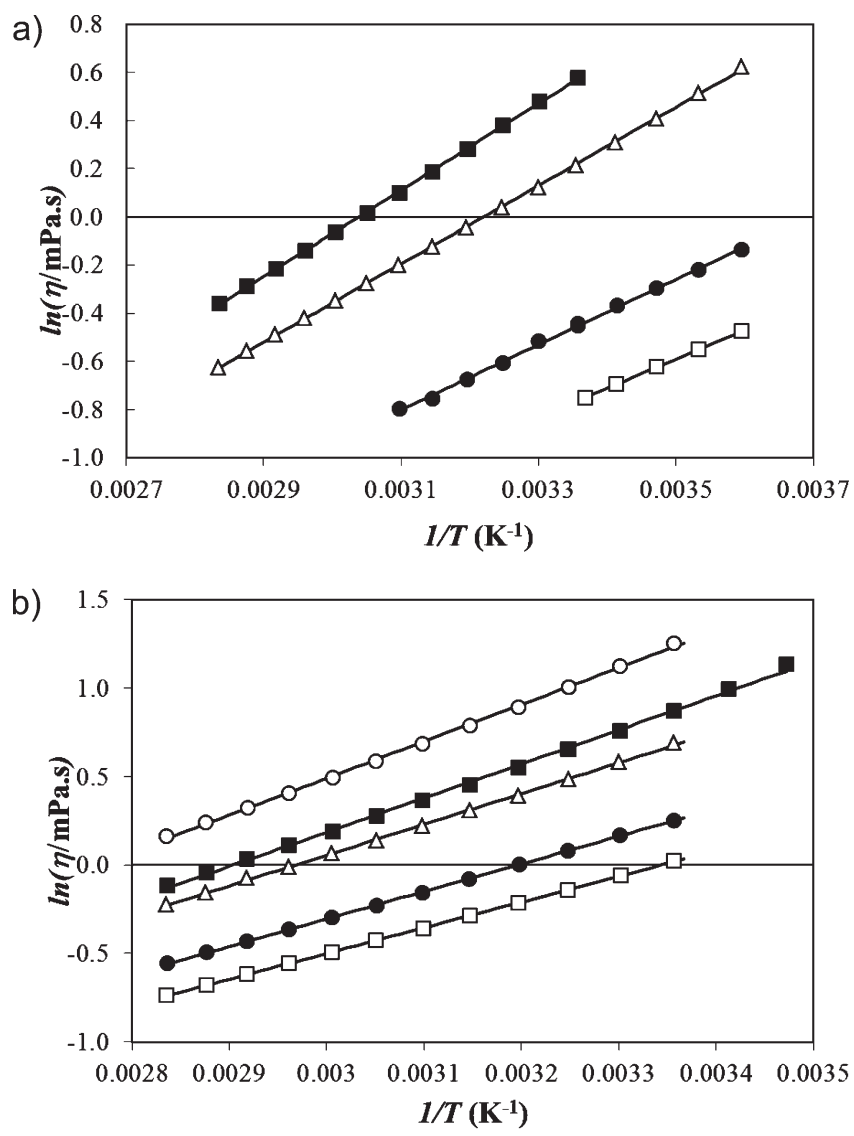

Figure 1. Logarithm of the viscosity as a function of the inverse temperature for: (a) perfluoroalkanes: F5 $(\square)$, F6 $(\bullet)$, F8 $(\triangle)$, F9 $(\boldsymbol{\square})$; (b) perfluoroalkylalkanes: F4H5 $(\square)$, F4H6 $(\bullet)$, F4H8 $(\triangle)$, F6H6 $(\boldsymbol{\square})$, F6H8 $(\bigcirc)$. The lines correspond to the fitting curves obtained from the Andrade equation.

compared at the same overall chain length. In the case of perfluoroalkylalkanes with 12 carbon atoms, it can be seen that the viscosities of the perfluoroalkylalkanes with the longest hydrogenated chain $(\mathrm{F} 4 \mathrm{H} 8)$ are closer to those for the corresponding $n$-alkane (n-dodecane) results than the other perfluoroalkylalkanes (F6H6).

As a first attempt to interpret the viscosity results for the semifluorinated compounds, a simple scheme that sums the contribution to the viscosity of the individual $\mathrm{CH}_{2}, \mathrm{CH}_{3}, \mathrm{CF}_{2}$,

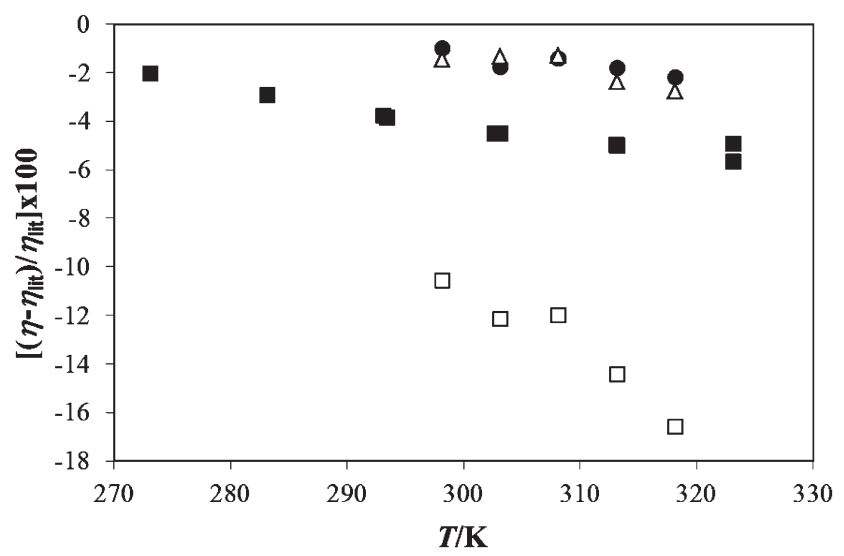

Figure 2. Relative deviations of the viscosity values reported herein with those for F6 from ref $50(\square)$, F6 from ref $52(\square)$, F8 from ref $50(\bullet)$, and F9 from ref $50(\triangle)$.

and $\mathrm{CF}_{3}$ groups in each PFAA molecule was developed. These contributions were estimated from the viscosity results for perfluoroalkanes and from literature results for $n$-alkanes. The differences between the experimental and estimated data should reflect the effect of mixing hydrogenated and fluorinated segments and the presence of the $\mathrm{CH}_{2}-\mathrm{CF}_{2}$ chemical bond. The procedure used to estimate the viscosities of perfluoroalkylalkanes was as follows: As described above, we first correlated the viscosity of perfluoroalkanes as a function of temperature using the Andrade equation, which allows, by interpolation, the values of viscosities at rounded temperatures to be calculated. For the $n$-alkanes the National Institute of Standards and Technology (NIST) correlations ${ }^{78}$ were used to determine the viscosities of $n$-pentane to $n$-dodecane, (excluding $n$-undecane) at temperatures from 273 to $373 \mathrm{~K}(273-310 \mathrm{~K}$ for $n$-pentane; $273-342 \mathrm{~K}$ for $n$-hexane; $273-373 \mathrm{~K}$ for $n$-heptane). At each temperature, linear correlations of $\ln \eta$ as a function of the number of $\mathrm{CH}_{2}$ or $\mathrm{CF}_{2}$ groups were calculated. The slope was interpreted as the $\mathrm{CH}_{2}$ (or $\mathrm{CF}_{2}$ ) increment for $\ln \eta$ and the intercept as twice the $\mathrm{CH}_{3}$ (or $\mathrm{CF}_{3}$ ) contribution. Each pair of parameters was then correlated with temperature. For hydrogenated segments, two linear fitting equations (one for each segment) were thus obtained expressing the dependence of the $\mathrm{CH}_{2}$ and $\mathrm{CH}_{3}$ group contribution on temperature. For $\mathrm{CF}_{2}$ and $\mathrm{CF}_{3}$, the corresponding increments were found to have a quadratic dependence on temperature and were therefore fitted to a second-order polynomial (Table 4). The viscosity of the PFAAs at each temperature was then obtained as the sum of the contribution of each type of segment multiplied by its frequency in the molecule. The calculated results following this procedure are compared with the experimental data in Figure 4. Simple averages of the deviations over the considered temperature range are shown in Table 5. From the observation of Figure 4 it can be seen that the scheme overestimates the viscosity of the PFAAs, that is, real substances are less viscous than the model predicts. This is not surprising since the model assumes ideal mixing of the alkyl and perfluoroalkyl segments. As seen in Table 5, the deviations between calculated and experimental values increases in the order F4H5 < F4H6 < F6H6 < F4H8 < F6H8, which can be attributed to two terms: a nonideal contribution to the viscosity from hypothetically mixing the hydrogenated and fluorinated segments that form the molecule and the effect introduced by the chemical bond linking the two types of segments. 

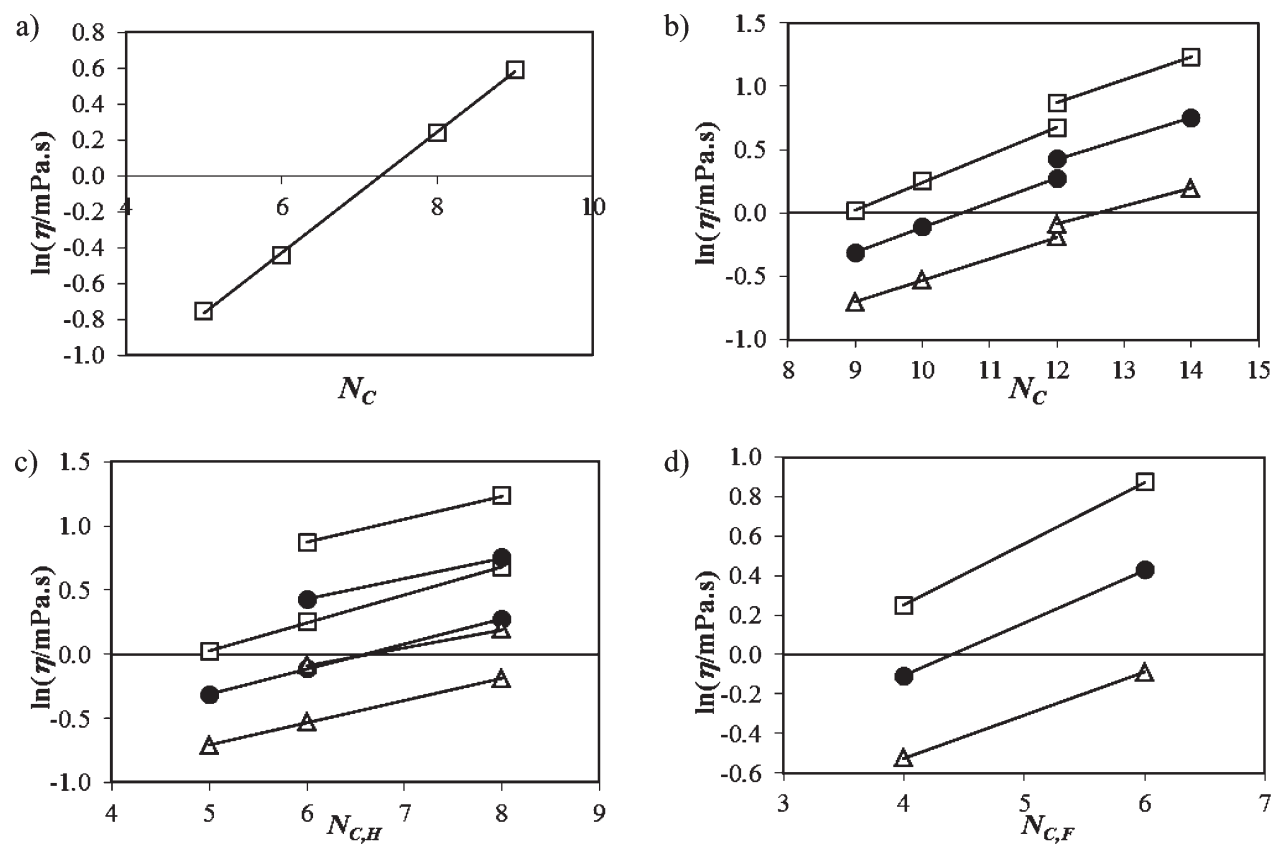

Figure 3. Interpolated values of logarithm of viscosity for: (a) perfluoroalkanes at $297 \mathrm{~K}$ (F5, F6, F8, and F9) as a function of chain length along with their respective linear fittings; semifluorinated alkanes (F4H5, F4H6, F4H8, F6H6, and F6H8) at 298, 320, and $350 \mathrm{~K}$ as a function of (b) chain length $\left(N_{\mathrm{C}}\right)$; (c) number of "hydrogenated" carbon atoms $\left(N_{\mathrm{C}, \mathrm{H}}\right)$; (d) number of "fluorinated carbon atoms" ( $N_{\mathrm{C}, \mathrm{F}}$, just F4H6 and F6H6).

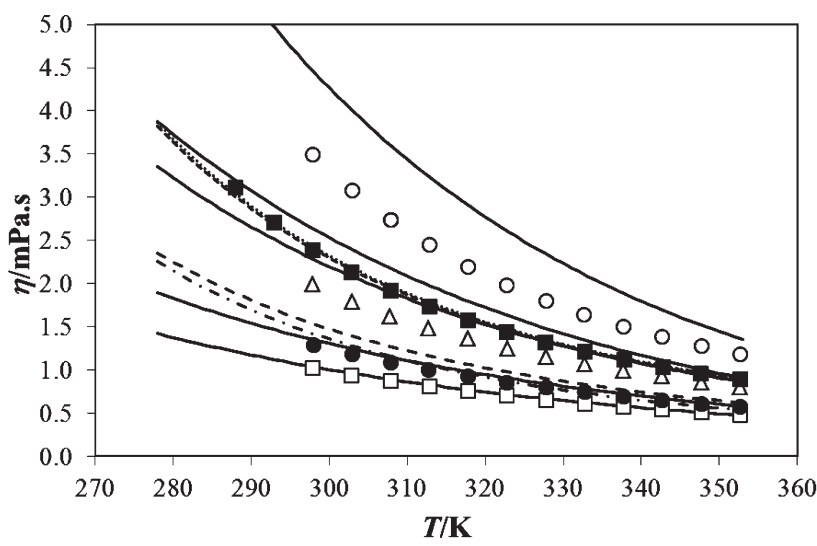

Figure 4. Viscosities of perfluoroalkylalkanes as a function of temperature. Experimental data: F4H5 $(\square)$, F4H6 $(\bullet)$, F4H8 $(\triangle)$, F6H6 (ם), F6H8 (O). Estimated: (solid lines). Sastri-Rao group contribution method: F4H5 (dotted-dashed line), F4H6 (long dashed line), F4H8 (short dashed line), F6H6 (dotted line).

Table 4. Group Contributions for the Logarithm of Viscosity as a Function of Temperature $\left[\ln \left(\eta_{i}\right)=a_{i}+b_{i} T+c_{i} T^{2}\right]$

\begin{tabular}{lccll} 
& $a_{i}$ & $b_{i}$ & \multicolumn{1}{c}{$c_{i}$} & std dev \\
$\mathrm{CH}_{3}$ & -0.56 & -0.019 & 0 & 0.0110 \\
$\mathrm{CH}_{2}$ & 0.603 & -0.00114 & 0 & 0.0018 \\
$\mathrm{CF}_{3}$ & 5.3 & -0.037 & $5.4 \times 10^{-5}$ & 0.0093 \\
$\mathrm{CF}_{2}$ & -0.23 & 0.0052 & $-1.11 \times 10^{-5}$ & 0.0026 \\
\hline
\end{tabular}

Estimating the nonideality contribution is quite a difficult task given the scarcity of viscosity data for mixtures of alkanes and perfluoroalkanes. For F6H6, however, an approximate estimation can be obtained from viscosity results for $(n$-hexane + perfluoroalkane) mixtures. ${ }^{79}$ At $298 \mathrm{~K}$, an equimolar mixture of $n$-hexane and perfluorohexane shows an excess viscosity (defined as $\eta^{\mathrm{E}}=\eta_{\mathrm{m}}-$ $x_{1} \eta_{1}-x_{2} \eta_{2}$, where $\eta_{\mathrm{m}}$ is the mixture viscosity and $\eta_{1}$ and $\eta_{2}$ are the viscosities of each of the pure components) of $\eta^{\mathrm{E}}=-0.0584 \mathrm{mPa} \cdot \mathrm{s}$, which corresponds to a reduction of $\sim 14 \%$ in the absolute viscosity. In the absence of additional experimental data, we can assume that the contribution of nonideality to the viscosity of $\mathrm{F} 6 \mathrm{H} 6$ will also result in a $14 \%$ decrease in the viscosity, that is, $-0.3439 \mathrm{mPa} \cdot \mathrm{s}$. Since the deviation between experimental and estimated viscosities for $\mathrm{F} 6 \mathrm{H} 6$ is $-0.2412 \mathrm{mPa} \cdot \mathrm{s}$, we thus conclude that the effect of the $\mathrm{CH}_{2}-\mathrm{CF}_{2}$ bond is positive (increases the viscosity and of the order of $0.1 \mathrm{mPa} \cdot \mathrm{s}$ ). This can be interpreted as follows: in the mixtures, the decrease of viscosity can be explained assuming that unlike segments glide rapidly over one another, given the weakness of the unlike intermolecular forces; in the PFAA, bonding the two moieties together implies that each segment always drags, attached to it, an unlike segment, which will obviously slow down the movement, thus increasing the viscosity. Furthermore, PFAAs possess a dipole at the $\mathrm{CH}_{2}-\mathrm{CF}_{2}$ junction that can be expected to increase cohesive forces and thus the viscosity.

We have also applied the Sastri-Rao viscosity estimation method $^{80}$ to obtain the viscosities of the PFAAs studied. This is a group-contribution approach, developed to predict the viscosity of pure liquids based on two empirical findings: the viscosity of pure liquids is inversely proportional to its vapor pressure on logarithmic scale and pure liquid viscosities at the normal boiling point temperatures are roughly constant for all the members of a given chemical family. In this method, the viscosities of pure liquids are determined by the equation:

$$
\eta=\eta_{\mathrm{B}} P_{\text {vap }}^{-N}
$$

where $P_{\text {vap }}$ is the vapor pressure of the liquid and $\eta_{\mathrm{B}}$ is the viscosity at the normal boiling point. The temperature dependence of the viscosity is thus accounted through the $P_{\text {vap }}$ versus $T$ curve, which, in principle, should be known. For temperatures 
below the boiling point, Sastri and Rao determine vapor pressure as a function of temperature with the equation:

$$
\begin{aligned}
\ln \left(p_{\text {vap }}\right)= & {\left[4.5398+1.0309 \ln \left(T_{\mathrm{B}}\right)\right] \times\left[1-\frac{\left(3-\left(2 T / T_{\mathrm{B}}\right)\right)^{0.19}}{T / T_{\mathrm{B}}}\right.} \\
& \left.-0.38\left(3-\left(2 T / T_{\mathrm{B}}\right)\right)^{-0.81} \ln \left(T / T_{\mathrm{B}}\right)\right]
\end{aligned}
$$

Sastri and Rao prefer this equation, even when a more accurate vapor pressure versus temperature relation is available, probably for internal consistency of the method. ${ }^{80}$ Both $\eta_{\mathrm{B}}$ and $N$ are obtained by group contributions as:

$$
\begin{gathered}
\eta_{\mathrm{B}}=\sum \Delta \eta_{\mathrm{B}}+\sum \Delta \eta_{\mathrm{B}, \text { corr }} \\
N=0.2+\sum \Delta N+\sum \Delta N_{\text {corr }}
\end{gathered}
$$

where $\Delta \eta_{\mathrm{B}}, \Delta N, \Delta \eta_{\mathrm{B}, \text { corr, }}$ and $\Delta N_{\text {corr }}$ are group-dependent contributions whose values per group are given in ref 80 . The contributions of the functional groups to $\eta_{\mathrm{B}}$ and $N$ are generally cumulative, except for $N$ when the compound contains more than one identical functional group. In that case, the contribution is taken only once, unless otherwise recommended.

The normal boiling point is therefore the key parameter in the application of the Sastri-Rao method; however, this quantity is not available in literature for the PFAAs under study. In the context of a systematic study on the thermodynamic properties of PFAAs, our research group has recently measured vapor pressures for $\mathrm{F} 4 \mathrm{H} 5, \mathrm{~F} 4 \mathrm{H} 6, \mathrm{~F} 4 \mathrm{H} 8$, and $\mathrm{F} 6 \mathrm{H} 6$ in a temperature range around room temperature. ${ }^{81}$ Normal boiling point temperatures were therefore obtained from these results by extrapolation, assuming the validity of the Clausius-Clapeyron equation. The Sastri-Rao method was then applied to all the PFAAs studied, except F6H8, for which no vapor pressure data was available. Equation 5 was used to ensure the self-consistency of the method. The results are shown in Figure 4 and Table 5. From the figure, it is apparent that for F4H5, $\mathrm{F} 4 \mathrm{H} 6$, and $\mathrm{F} 4 \mathrm{H} 8$ the estimated values are systematically higher than

Table 5. Average Relative Deviations between Experimental and Estimated Viscosities over All Temperatures for the Studied Semifluorinated Alkanes

\begin{tabular}{cc} 
compound & $\begin{array}{c}\text { average \% deviations over } \\
\text { the whole temperature range }\end{array}$ \\
F4H5 & 1.8 \\
F4H6 & 4.7 \\
F4H8 & 14.4 \\
F6H6 & 11.4 \\
F6H8 & 27.5 \\
\hline
\end{tabular}

the experimental ones and higher than those obtained by the additive scheme previously described. The average relative deviations from experimental results are $\sim 10 \%, \sim 14 \%$, and $17 \%$, respectively, for F4H5, F4H6, and F4H8. For F6H6, however, the method predicts the viscosity as a function of temperature with an average relative deviation of $\sim 2 \%$. It seems that the deviations increase with the asymmetry of the PFAA molecule in terms of the number of "fluorinated" and "hydrogenated" carbon atoms. We note that the Sastri-Rao method is able to predict the viscosity of $n$-alkanes and perfluoroalkanes with relative deviations up to $\pm 5 \%$, in the same temperature range used in this work. However, it should be emphasized that the quality of the estimations of this method is strongly dependent on the accuracy of the boiling point value used, which in this case was obtained by extrapolating the low pressure portions of $P_{\text {vap }}$ versus $T$ curves.

Finally, to obtain a more molecular-level understanding of the viscosity behavior of PFAA molecular dynamics simulations were performed to predict the viscosity using a published all-atom force field as described in Section 3. Liquid densities were calculated for each of the PFAA molecules at atmospheric pressure and $298.15 \mathrm{~K}$ to verify that the force field predicts the correct values and can be used in the subsequent viscosity calculations. The results are presented in Table 6, from which we note that the densities are smaller than the experimental data by $1.5-3.5 \%$. The results of the viscosity calculations are presented in Figure 5, and the average value of the viscosity is also reported in Table 6 . In agreement with the experimental data the viscosity increases as the proportion of hydrocarbon and fluorocarbon in the molecules is increased (i.e., the viscosity increases $\mathrm{F} 4 \mathrm{H} 5<\mathrm{F} 4 \mathrm{H} 6<\mathrm{F} 4 \mathrm{H} 8$ and $\mathrm{F} 6 \mathrm{H} 6<\mathrm{F} 6 \mathrm{H} 8$ ) and is greater for molecules of equal chain length but a higher fluorocarbon fraction than hydrocarbon (i.e., the viscosity of F4H8 < F6H6).

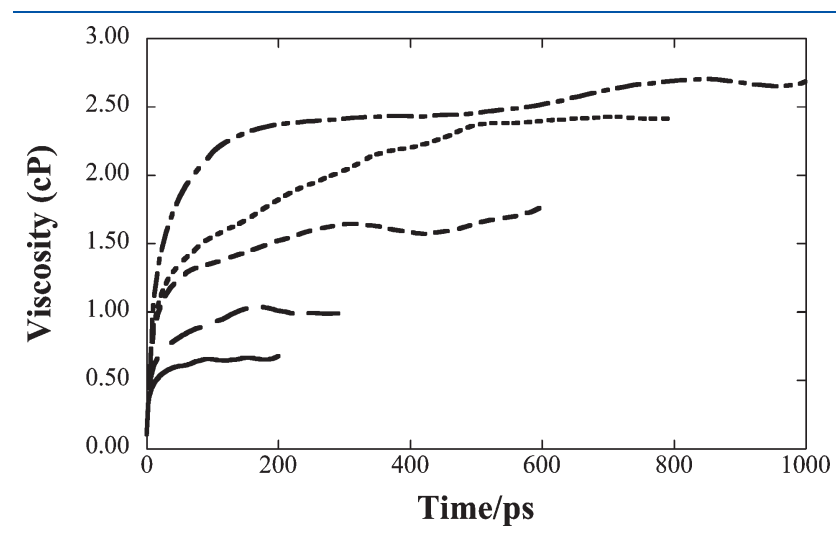

Figure 5. Viscosities at $298.15 \mathrm{~K}$ calculated from equilibrium molecular dynamics simulations. Solid line corresponds to F4H5, long dashed to

\begin{tabular}{|c|c|c|c|c|c|c|c|}
\hline \multirow[b]{2}{*}{ compound } & \multirow[b]{2}{*}{$\tau / \mathrm{ps}$} & \multicolumn{3}{|c|}{$\operatorname{density}\left(\rho / \mathrm{kg} \cdot \mathrm{m}^{-3}\right)$} & \multicolumn{3}{|c|}{ viscosity $(\eta / \mathrm{mPa} \cdot \mathrm{s})$} \\
\hline & & simulation & experiment & \%deviation & simulation & experiment & \%deviation \\
\hline F4H5 & 68.4 & 1241 & 1286.95 & -3.57 & $0.66 \pm 0.01$ & 1.015 & -35.4 \\
\hline F4H6 & 125 & 1224 & 1257.61 & -2.67 & $1.00 \pm 0.02$ & 1.281 & -22.1 \\
\hline F4H8 & 300 & 1182 & 1209.08 & -2.24 & $1.64 \pm 0.05$ & 1.957 & -16.3 \\
\hline F6H6 & 364 & 1351 & 1386.36 & -2.55 & $2.36 \pm 0.01$ & 2.384 & -1.0 \\
\hline F6H8 & 776 & 1313 & 1329.88 & -1.27 & $2.65 \pm 0.05$ & 3.416 & -22.4 \\
\hline
\end{tabular}
F4H6, short dashed to F4H8, dots to F6H6 and dash-dot to F6H8.

Table 6. Results of Rotational Relaxation $(\tau)$, Density, and Viscosity Calculations for PFAAs Studied from Molecular Dynamics Simulation at 298.15 K and Comparison with Experimental Results 
This behavior is also in agreement with previous observations that fluorocarbon chains are more rigid than hydrocarbon chains and so generally exhibit higher viscosities. ${ }^{9,58}$ As can be seen from the table, the simulations consistently underestimate the viscosity with deviations of $15-35 \%$. It should be noted, however, that the simulations were performed at slightly lower densities and that deviations of this order are not unusual when comparing experimental and simulated viscosities for $n$-alkanes and $n$-perfluoroalkanes. ${ }^{82,83}$ The deviation observed could also be due to the nonideality of alkane-perfluoroalkane interactions, in particular, for the $\mathrm{H}-\mathrm{F}$ interaction. As previously explained, it has been demonstrated that simple geometric or Lorentz-Berthelot combining rules are typically unable to describe the behavior of mixtures involving alkanes and perfluoroalkanes, irrespective of the level of detail of force field used. Given the observed agreement with experiment for the density, it seems that to obtain accurate predictions of the viscosity additional changes to the force field other than simply fitting the cross interaction energy will be required.

\section{CONCLUSIONS}

Experimental viscosity data at atmospheric pressure are reported for four perfluoroalkanes and five perfluoroalkylalkanes, in the temperature range from 278 to $353 \mathrm{~K}$. The results for all systems follow an Arrhenius-like trend. The perfluoroalkylalkanes display viscosities that are intermediate between those of the $n$-alkanes and the $n$-perfluoroalkanes with the same chain length.

The experimental results were interpreted in terms of the contributions to the viscosity of the individual $\mathrm{CH}_{2}, \mathrm{CH}_{3}, \mathrm{CF}_{2}$, and $\mathrm{CF}_{3}$ groups in each PFAA molecule. These were estimated from the viscosity results for perfluoroalkanes and from literature results for $n$-alkanes. The calculated values overestimate the experimental results for all systems, and the deviations were rationalized as resulting from the nonideal mixing of alkane and perfluoroalkane segments within the molecule and the presence of the $\mathrm{CF}_{2}-\mathrm{CH}_{2}$ junction. Using experimental viscosity data for the $(n$-hexane + perfluorohexane) mixture, a positive value of $\sim 0.1 \mathrm{mPa} \cdot \mathrm{s}$ was estimated for the junction contribution in F6H6. A standard group contribution method (Sastri-Rao) was also used to estimate the viscosities of the perfluoroalkylalkanes studied and produced consistently positive deviations that seem to increase with the fluorinated/hydrogenated asymmetry of the molecule. Viscosities were also predicted from molecular dynamics simulations for each PFAA studied at a single temperature, using a force field taken from the literature. In all cases the simulation results are found to be smaller than the experimental ones, though the deviations are much smaller for F6H6 than the other molecules studied.

\section{ACKNOWLEDGMENT}

P.M. acknowledges funding from Fundação para Ciência e Tecnologia, in the form of a Ph.D. grant (No. SFRH/BD/39150/ 2007). E.J.M.F. acknowledges funding from the Fundação para Ciência e Tecnologia through Grant No. POCI/QUI/61850/2004. L.F.G.M. and C.M.C.L. acknowledge funding from the Fundação para Ciência e Tecnologia through Grant No. POCTI/QUI/ 46299/2002. C.M.C. and J.B.L. acknowledge support from the Office of Naval Research under Grant Nos. N00014-06-1-0624, N00014-09-1-0334, and N00014-09-10793 and gratefully acknowledge the National Energy Research Scientific Computing Center, which is supported by the Office of Science of the Department of Energy under Contract No. DE-AC02-05CH11231, for computational resources. C.M.C. also acknowledges support from the Jacob Wallenberg Foundation.

\section{REFERENCES}

(1) Gladysz, J. A.; Curran, D. P. Tetrahedron 2002, 58, 3823.

(2) Horvath, I. T.; Rabai, J. Science 1994, 266, 72.

(3) Eckert, C. A.; Knutson, B. L.; Debenedetti, P. G. Nature 1996, 383, 313.

(4) McClain, J. B.; Betts, D. E.; Canelas, D. A.; Samulki, E. T.; De Simone, J. M.; Londono, J. D.; Cochran, H. D.; Wignall, G. D.; ChilluraMartino, D.; Triolo, R. Science 1996, 274, 2049.

(5) May, G. Chem. Br. 1997, 33, 34.

(6) Riess, J. G. J. Fluorine Chem. 2002, 114, 119.

(7) Riess, J. G. Chem. Rev. 2001, 101, 2797.

(8) Dias, A. M. A.; Gonçalves, C. M. B.; Caço, A. I.; Santos, L. M. N. B. F.; Piñeiro, M. M.; Vega, L. V.; Coutinho, J. A. P.; Marrucho, I. M. J. Chem. Eng. Data 2005, 50, 1328.

(9) Jang, S. S.; Blanco, M.; Goddard, W. A., III; Caldwell, G.; Ross, R. B. Macromolecules 2003, 36, 5331.

(10) Song, W.; Rossky, P. J.; Maroncelli, M. J. Chem. Phys. 2003, 119,9145 .

(11) Scott, R. L. J. Phys. Chem. 1958, 62, 136.

(12) Duce, C.; Tinè, M. R.; Lepori, L.; Matteoli, E. Fluid Phase Equilib. 2002, 199, 197.

(13) Pratas de Melo, M. J.; Dias, A. M. A.; Blesic, M.; Rebelo, L. P. N.; Vega, L. F.; Coutinho, J. A. P.; Marrucho, I. M. Fluid Phase Equilib. 2006, 242, 210.

(14) Lepori, L.; Matteoli, E.; Spanedda, A.; Duce, C.; Tinè, M. R. Fluid Phase Equilib. 2002, 201, 119.

(15) Morgado, P.; Tomás, R.; Zhao, H.; dos Ramos, M. C.; Blas, F.J.; McCabe, C.; Filipe, E. J. M. J. Phys. Chem. C 2007, 111, 15962.

(16) Morgado, P.; Rodrigues, H.; Blas, F. J.; McCabe, C.; Filipe, E. J. M. Fluid Phase Equilib. 2011, 306, 76.

(17) Hildebrand, J. H.; Prausnitz, J.; Scott,R. Regular and Related Solutions: The Solubility of Gases, Liquids, and Solids; van Nostrand Reinhold: New York, 1970.

(18) McCabe, C.; Galindo, A.; Gil-Villegas, A.; Jackson, G. J. Phys. Chem. B 1998, 102, 8060.

(19) Morgado, P.; McCabe, C.; Filipe, E. J. M. Fluid Phase Equilib. 2005, 228-229, 389.

(20) Colina, C. M.; Galindo, A.; Blas, F. J.; Gubbins, K. E. Fluid Phase Equilib. 2004, 222, 77.

(21) Colina, C. M.; Gubbins, K. E. J. Phys. Chem. B 2005, 109, 2899.

(22) de Melo, M. J. P.; Dias, A. M. A.; Blesic, M.; Rebelo, L. P. N.; Vega, L. F.; Coutinho, J. A. P.; Marrucho, I. M. Fluid Phase Equilib. 2006, $242,210$.

(23) Aparicio, S. J. Supercrit. Fluids 2008, 46, 10.

(24) Varanda, F. R.; Vega, L. F.; Coutinho, J. A. P.; Marrucho, I. M. Fluid Phase Equilib. 2008, 268, 85.

(25) Peguin, R. P. S.; Kamath, G.; Potoff, J. J.; da Rocha, S. R. P. J. Phys. Chem. B 2009, 113, 178.

(26) Watkins, E. K.; Jorgensen, W. L. J. Phys. Chem. A 2001, 105,4118

(27) Potoff, J. J.; Bernard-Brunel, D. A. J. Phys. Chem. B 2009, 113,14725 .

(28) Amat, M. A.; Rutledge, G. C. J. Chem. Phys. 2010, 132, 9.

(29) McCabe, C.; Bedrov, D.; Borodin, O.; Smith, G. D.; Cummings, P. T. Ind. Eng. Chem. Res. 2003, 42, 6956.

(30) McCabe, C.; Bedrov, D.; Smith, G. D.; Cummings, P. T. Ind. Eng. Chem. Res. 2001, 40, 473.

(31) Zhang, L.; Siepmann, J. I. J. Phys. Chem. B 2005, 109, 2911.

(32) Cui, S. T.; Siepmann, J. I.; Cochran, H. D.; Cummings, P. T. Fluid Phase Equilib. 1998, 146, 51.

(33) Cui, S. T.; Cochran, H. D.; Cummings, P. T. J. Phys. Chem. B 1999, 103, 4485.

(34) Turberg, M. P.; Brady, J. E. J. Am. Chem. Soc. 1998, 110, 7797. 
(35) Binks, B. P.; Fletcher, P. D. I.; Kotsev, S. N.; Thompson, R. L. Langmuir 1997, 13, 6669.

(36) Viney, C.; Russell, T. P.; Depero, L. E.; Twieg, R. J. Mol. Cryst. Liq. Cryst. 1989, 168, 63.

(37) Viney, C.; Twieg, R. J.; Russell, T. P.; Depero, L. E. Liq. Cryst. 1989, 5, 1783.

(38) Maaloum, M.; Muller, P.; Krafft, M. P. Angew. Chem., Int. Ed. 2002, 41, 4331.

(39) Simões Gamboa, A. L.; Filipe, E. J. M.; Brogueira, P. Nano Lett. 2002, 2, 1083.

(40) Rabolt, J. F.; Russell, T. P.; Twieg, R. J. Macromolecules 1984, 17, 2786.

(41) Russell, T. P.; Rabolt, J. F.; Twieg, R. J.; Siemens, R. L.; Farmer, B. L. Macromolecules 1986, 19, 1135.

(42) Höpken, J.; Möller, M. Macromolecules 1992, 25, 2482.

(43) Marczuk, P.; Lang, P. Macromolecules 1998, 31, 9013.

(44) Riess, J. G. Artif. Cell Blood Substit. Biotechnol. 2006, 34, 567.

(45) Bertilla, S. M.; Thomas, J. L.; Marie, P.; Krafft, M. P. Langmuir 2004, 20, 3920.

(46) Schutt, E. G.; Klein, D. H.; Mattrey, R. M.; Riess, J. G. Angew. Chem. 2003, 115, 3336.

(47) Lindner, J. R. Nat. Rev. Drug Discovery 2004, 3, 527.

(48) Klibanov, A. L. Invest. Radiol. 2006, 41, 354.

(49) Shchukin, D. G.; Köhler, K.; Möhwald, H.; Sukhorukov, G. B. Angew. Chem. 2005, 117, 3375.

(50) Gerber, F.; Waton, G.; Krafft, M. P.; Vandamme, T. F. Artif. Cells Blood Substit. Immobil. Biotechnol. 2007, 35, 119.

(51) Rossi, S.; Waton, G.; Krafft, M. P. ChemPhysChem 2008, 9, 1982.

(52) de Loos, T. W.; Poot, W. Int. J. Thermophys. 1988, 19, 637.

(53) Tochigi, K.; Satou, T.; Kurihara, K.; Ochi, K.; Yamamoto, H.; Mochizuki, Y.; Sako, T. J. Chem. Eng. Data 2001, 46, 913.

(54) Morgado, P.; Zhao, H.; Blas, F. J.; McCabe, C.; Rebelo, L. P. N.; Filipe, E. J. M. J. Phys. Chem. B 2007, 111, 2856.

(55) Morgado, P., submitted for publication.

(56) McCabe, C.; Gil-Villegas, A.; Jackson, G.; Del Rio, F. Mol. Phys. 1999, 97, 551.

(57) Peng, Y.; Zhao, H.; McCabe, C. Mol. Phys. 2006, 104, 571.

(58) Hariharan, A.; Harris, J. G. J. Chem. Phys. 1994, 101, 4156.

(59) Friedemann, R.; Naumann, S.; Brickmann, J. Phys. Chem. Chem. Phys. 2001, 3, 4195.

(60) Escobedo, F. A. J. Chem. Phys. 2004, 121, 11463.

(61) Pádua, A. A. H. J. Phys. Chem. A 2002, 106, 10116.

(62) Deschamps, J.; Costa Gomes, M. F.; Pádua, A. A. H. J. Fluorine Chem. 2004, 125, 409.

(63) Morgado, P., to be published.

(64) Pierce, F.; Tsige, M.; Borodin, O.; Perahia, D.; Grest, G. S. J. Chem. Phys. 2008, 128, 214903.

(65) Freire, M. G.; Ferreira, A. G. M.; Fonseca, I. M. A.; Marrucho, I. M.; Coutinho, J. A. P. J. Chem. Eng. Data 2008, 53, 538.

(66) McCabe, C.; Cui, S. T.; Cummings, P. T. Fluid Phase Equilib. 2001, 183, 363-370.

(67) McCabe, C.; Cui, S. T.; Cummings, P. T.; Gordon, P. A.; Saeger, R. B. J. Chem. Phys. 2001, 114, 1887-1891.

(68) Bair, S.; McCabe, C.; Cummings, P. T. Phys. Rev. Lett. 2002, 88 , article no. 058302.

(69) Jorgensen, W. L.; Maxwell, D. S.; Tirado Rives, J. J. Am. Chem. Soc. 1996, 118, 11225.

(70) Watkins, E. K.; Jorgensen, W. L. J. Phys. Chem. A 2001, 105, 4118.

(71) Plimpton, S. J. Comput. Phys. 1995, 117, 1.

(72) Tuckerman, M.; Berne, B. J.; Martyna, G. J. J. Chem. Phys. 1992, 97, 1990.

(73) Daivis, P. J.; Evans, D. J. J. Chem. Phys. 1994, 100, 541.

(74) Allen, M. P., Tildesley, D. J. Computer Simulation of Liquids; Oxford University Press: Oxford, 1987.

(75) Mondello, M.; Grest, G. S. J. Chem. Phys. 1997, 106, 9327.

(76) Gordon, P. A. Ind. Eng. Chem. Res. 2003, 42, 7025.

(77) Stiles, V. E.; Cady, G. H. J. Am. Chem. Soc. 1952, 74, 3771.
(78) Lemmon, E. W.; McLinden, M. O.; Friend, D. G. Thermophysical Properties of Fluid Systems. In NIST Chemistry WebBook, NIST Standard Reference Database Number 69; Linstrom, P. J., Mallard, W. G., Eds.; National Institute of Standards and Technology: Gaithersburg, MD; http://webbook.nist.gov (retrieved Jan 20, 2011).

(79) Morgado, P., to be published

(80) Sastri, S. R. S.; Rao, K. K. Chem. Eng. J. 1992, 50, 9.

(81) Morgado, P., to be published.

(82) Martin, M. G.; Thompson, A. P. Fluid Phase Equilib. 2004, 217, 105.

(83) Zhang, H.; Ely, J. F. Fluid Phase Equilib. 2004, 217, 111. 